\title{
EFEITO DE EXTRATOS VEGETAIS NO CONTROLE DE Euschistus heros (F.) (HEMIPTERA: PENTATOMIDAE) EM LAVOURA DE SOJA NA REGIÃO SUDOESTE DO ESTADO DE MATO GROSSO
}

\author{
Valvenarg Pereira da Silva ${ }^{1}$, Mônica Josene Barbosa Pereira ${ }^{1}$, Leonardo Morais Turchen ${ }^{1}$ \\ ${ }^{1}$ Universidade do Estado de Mato Grosso/UNEMAT - Tangará da Serra, MT, E-mail: silvabiologo@hotmail.com, \\ monica@unemat.br
}

\section{RESUMO}

Esta pesquisa teve como objetivo avaliar a eficiência dos extratos vegetais de Annona coriacea, Annona crassiflora e do produto comercial à base de óleo de nim sobre o percevejo Euchistus heros em lavoura de soja. Para isso, o experimento foi conduzido em delineamento de blocos casualizados com cinco tratamentos e quatro repetições. Os tratamentos foram $\mathrm{T} 1=$ extrato de $A$. coriacea $(2 \%), \mathrm{T} 2=$ extrato de $A$. crassiflora $(2 \%), \mathrm{T} 3=$ Controle 1 (Dimetilsulfóxido a 20\%), T4 = Produto à base de nim, T5 = Controle 2 (Água). A aplicação dos produtos foi realizada quando o nível de controle atingiu 2 percevejos/pano de batida. Assim, para avaliar a eficiência dos tratamentos foram efetuadas amostragens da população de E. heros antes das aplicações dos inseticidas e 2, 5 e 7 dias após. Com isso, as avaliações indicaram que ao sétimo dia após as aplicações os extratos vegetais e o produto comercial à base de nim obtiveram eficiência no controle da população de E. heros, diferindo estatisticamente das testemunhas (Água e DMSO 20\%), em que o extrato de $A$. coriacea obteve $26,73 \%$ de eficiência, $A$. crassiflora $16,83 \%$ e o produto à base de nim $24,76 \%$ de eficiência. Tais resultados indicam que os extratos vegetais podem se tornar uma alternativa promissora para o controle desta praga no campo, mas para isso são necessárias pesquisas complementares que avaliem por períodos mais longos estes produtos, com o intuito de se obter maior eficiência.

Palavras-chave: Annonaceae, pragas, fitoinseticida, Meliaceae, Euchistus heros

\section{EFFECT OF PLANT EXTRACTS ON THE CONTROL OF Euschistus heros (F.) (Hemiptera: Pentatomidae) ON SOYBEAN CROP IN THE SOUTHWESTERN REGION OF MATO GROSSO STATE, BRAZIL}

\section{ABSTRACT}

This research aimed to evaluate the efficiency of Annona coriacea and A. crassiflora extracts and neem based product on brown stink bug, Euschistus heros in soybean crop. The experiment was conducted in randomized block design with five treatments and four replications. The treatments were: $\mathrm{T} 1=$ A coriace extract, $\mathrm{T} 2=\mathrm{A}$. crassiflora extract, $\mathrm{T} 3=$ Control $1(20 \%$ Dimethylsulfoxide), T4 = neem based product and T5 = Control (water). The application of the treatments performed when the population of stink bugs reached the level of control (2 Stink bug/beat). To evaluate the efficiency of treatments one performed samplings with 2, 5 and 7 days after application, using five cloth beat per plot. The evaluations indicated that at the seventh day after applications, extracts and neem based product showed efficiency in population control of $E$. heros and statistically differed from controls (water and DMSO 20\%). The A. coriacea extract had $26.73 \%$ of efficiency, A. crassiflora extract $16.83 \%$ and neem based product $24.76 \%$ of control efficiency. These results indicated that plant extract may become a promising alternative for pest control in field, however more research is necessary to evaluated these products for longer periods, in order to obtain higher, efficiency.

Keywords: Annonaceae, pest, phytoinsecticide, Meliaceae, Euchistus heros 


\section{INTRODUÇÃO}

O percevejo Euschistus heros (Fabricius, 1798) é considerado praga chave nas lavouras de soja do estado de Mato Grosso (VIVAN \& DEGRANDE, 2011).

Este inseto se alimenta diretamente dos grãos e vagens de soja tornando-as chochas e enrugadas, e isso reduz a produção e qualidade das sementes (PANIZZI \& SLANSKY JUNIOR, 1985; PANIZZI et al., 2012). Os danos ocasionados por este percevejo são irreversíveis e, quando não controlados, as perdas econômicas podem chegar até $30 \%$ na produção (VIVAN \& DEGRANDE, 2011). Para se evitar estes prejuízos, o controle deste percevejo é realizado exclusivamente através de aplicações de inseticidas químicos (CORRÊAA-FERREIRA et al., 2010). Contudo, a utilização deste método tem ocasionado diversos problemas, tais como: contaminação ambiental e humana, seleção de populações de percevejos resistentes e redução de inimigos naturais (SOSAGOMEZ et al., 2001; BUENO et al., 2011).

Diante dos inúmeros problemas advindos do mal uso de inseticidas, estudos têm sido realizados em busca por estratégias alternativas para o manejo de pragas; dentre essas, o uso de extratos vegetais tem se destacado como fonte promissora de compostos com atividade inseticida (COSTA et al., 2004), principalmente porque apresentam baixa toxicidade e pouca persistência no ambiente, e podem ainda ser associadas com as demais estratégias de controle (CAVALCANTE et al., 2006).

Dentre as plantas com ação inseticida, Azadirachta indica (A. Juss) da família Meliaceae, popularmente conhecida como nim, tem mostrado resultados promissores no controle de percevejos pragas da soja. Pesquisa realizada por Peres \& Corrêa-Ferreira (2006), evidenciou a eficiência de produtos à base de nim, sobre ninfas de $3^{\circ}$ instar do percevejo E. heros, com mortalidade de $47,9 \%$ e $94,2 \%$ nas concentrações de 0,5 e $5,0 \%$, respectivamente em condições de laboratório. Em condições de campo na cultura de feijão, o produto comercial Neemix 4.5 EC à base de Azadirachtina, ocasionou a redução da densidade populacional do percevejo Nezara viridula (Linnaeus, 1758), após a sua aplicação durante 10 dias de avaliação (ABUDULAI $e t$ al., 2003). Além de ocasionar a mortalidade dos insetos, o extrato de nim também apresenta efeito anti-alimentar sobre os percevejos pragas da soja. Este efeito foi observado sobre o percevejo $N$. viridula, em nozes tratadas com o extrato de sementes de nim (SEYMOUR et al., 2005).

Plantas da família Annonaceae também surgem como alternativa promissora para o controle do percevejo E. heros, uma vez que possuem em sua composição acetogeninas, que apresentam potencial inseticida (JACOBSON, 1989; ALALI, et al., 1999). Com base nesse fato, pesquisas conduzidas em laboratório já evidenciaram a ação inseticida de extratos de anonáceas sobre os percevejos da soja. Souza et al. (2007) observaram mortalidade de $100 \%$ de ninfas de $3^{\circ}$ instar do percevejo Dichelops melacanthus (Dallas, 1851) com o extrato de Annona coriacea (Mart) nas concentrações 4 e $8 \%$. Além disso, Oliveira \& Pereira (2009) verificaram que o extrato de Annona crassiflora (Mart) possui efeito antialimentar sobre o percevejo E. heros, com redução de $51 \%$ na quantidade de pontos de alimentação em vagens de feijão.

Entretanto, apesar dos extratos de anonáceas apresentarem resultados promissores em condições de laboratório, pesquisas que demonstrem esta potencialidade em campo são incipientes. Isso restringe a identificação do potencial destes extratos no controle de pentatomídeos em lavouras da soja. Desta forma, este trabalho teve por objetivo avaliar a eficiência, em campo, dos extratos de $A$. 
coriacea, A. crassiflora e do produto comercial à base de nim sobre o percevejo $E$. heros.

\section{MATERIAL E MÉTODOS}

Esta pesquisa foi desenvolvida durante a safra sojícola 2011/12 na fazenda Aparecida da Serra, situada a $100 \mathrm{~km}$ do município de Tangará da Serra, MT, onde na área experimental $\left(14^{\circ} 18^{\prime} 59^{\prime}\right.$ 'S e $57^{\circ} 45^{\prime}$ 16"W) foi implantada a cultivar TMG 132 RR de ciclo semiprecoce, plantada com espaçamento entre plantas de $0,50 \mathrm{~m}$.

Para obtenção dos extratos vegetais das espécies de $A$. coriacea e $A$. crassiflora, frutos foram coletados em áreas de Cerrado no município de Campo Novo dos Parecis e Distrito de Deciolândia, MT, respectivamente. $\mathrm{O}$ processo de preparação dos extratos vegetais foi realizado no laboratório de Entomologia da Universidade do Estado de Mato Grosso, Campus de Tangará da Serra. As sementes de $A$. coriacea e A. crassiflora, foram dessecadas em estufa com circulação forçada de $40^{\circ} \mathrm{C}$, por 72 horas. Após este período, as sementes foram trituradas em moinho tipo faca, até a obtenção de um pó.

Em seguida o pó obtido no processo de trituração das sementes foi misturado com o solvente (álcool metílico), na proporção de $500 \mathrm{~g}$ de pó por $1500 \mathrm{~mL}$ solvente. A mistura foi mantida em recipiente de vidro hermeticamente fechado por sete dias para percolação. A suspensão foi filtrada em funil Büchner e posteriormente o solvente foi evaporado em rotavapor até adquirir a estabilidade de massa, obtendo, desta forma, o extrato bruto metanólico. A partir deste extrato foram feitas as diluições para as concentrações desejadas, utilizando como solubilizante dimetilsulfóxido (DMSO) a $20 \%$.

O delineamento experimental se deu em blocos casualizados com cinco tratamentos e quatro repetições. Cada bloco foi distribuído em uma área de $6.300 \mathrm{~m}^{2}$, distante $15 \mathrm{~m}$ entre si, totalizando 2,52 hectares. Nestes blocos foram distribuídas cinco parcelas de 10 x $10 \mathrm{~m}\left(100 \mathrm{~m}^{2}\right)$, contendo 20 fileiras de soja com espaçamento de $0,50 \mathrm{~m}$ cada parcela, distanciadas 15 metros entre si, nas quais foram distribuídos os seguintes tratamentos: $\mathrm{T} 1=$ Extrato de $A$. coriacea $(2 \%), \mathrm{T} 2=$ Extrato de A. crassiflora (2\%), T3= Controle 1 - DMSO (20\%), T4 = Produto a base de Nim (dosagem 2L/ha) T5 = Controle 2 (Água). Para aplicação do extrato, foi utilizada a metodologia adaptada de Batista Filho et al. (2003) e Ramiro et al. (2005).

Como área útil das parcelas foram utilizadas as 10 linhas centrais da soja, para avaliação dos tratamentos.

Para a realização das aplicações foram efetuadas amostragens prévias semanalmente com pano de batida, a fim de monitorar o nível populacional do percevejo nas parcelas. Quando a população atingiu o nível de controle ( 2 percevejos adultos/pano de batida) foi efetuada uma amostragem prévia, para avaliar a homogeneidade entre os tratamentos, e em seguida, efetuou-se as aplicações. Desse modo, os tratamentos foram aplicados com pulverizador de $\mathrm{CO}_{2}$ pressurizado, contendo quatro bicos do tipo cone com espaçamento de $0,50 \mathrm{~m}$ entre eles, com vazão de 200 L.ha $^{-1}$.

Para avaliar a eficiência dos tratamentos foram realizadas amostragens aos 2, 5, e 7 dias após a aplicação (DAA), a fim de estimar a densidade populacional de E. heros nos tratamentos. As avaliações foram realizadas sempre pela manhã, quando os percevejos se encontram na parte mais alta da planta. De acordo com Vivan \& Degrande (2011) a realização do pano de batida durante este período aumenta a eficiência na amostragem.

Os dados foram submetidos à análise de variância e, quando significativo, as médias foram agrupadas pelo teste de ScottKnott a 5\% de probabilidade de erro, através 
do programa estatístico SASM - Agri versão 4.0. A porcentagem de eficiência dos tratamentos foi calculada através da fórmula de Abbott (1925), que é dada por: $\% \mathrm{E}=(1$ $\mathrm{TI} / \mathrm{T}) \times 100, \mathrm{~T}=$ número de insetos vivos na testemunha e $\mathrm{TI}=$ número de insetos vivos no tratamento. Como existem dois controles, os valores de $\mathrm{T}$ para o cálculo de eficiência dos extratos de $A$. coriacea e A. crassiflora são obtidos do controle 1 (DMSO 20\%) e os valores de $\mathrm{T}$ para o cálculo de eficiência do produto à base de nim do controle 2 (água).

\section{RESULTADOS E DISCUSSÃO}

Os resultados da amostragem prévia indicaram a homogeneidade da população de percevejos entre os tratamentos $(\mathrm{F}=1,187 \mathrm{e}$ $p>0,05)$ e comprovaram que a população estava acima do nível de controle (2 percevejos/pano de batida), e isso cumpre o requisito para aplicação dos produtos.

$\mathrm{Na}$ amostragem aos 2 e 5 dias após aplicação não foi verificada diferença entre os tratamentos $(\mathrm{F}=1,21$ e $p>0,05 ; \mathrm{F}=0,33$ e $p>0,05$ ), assim indica que a população se manteve igual as testemunhas. Na avaliação ao sétimo dia após a aplicação houve diferença estatística entre os tratamentos $(\mathrm{F}=3,91$ e $p<0,05)$, nos quais todos os fitoinseticidas diferiram estatisticamente dos controles Água e DMSO 20\%, e estes não diferiram entre si. A eficiência de controle foi de $26,73 \%$ para extrato de A. coriacea, de $16,83 \%$ para o extrato de $A$. crassiflora e de $24,76 \%$ para o produto à base de nim (Tabela 1).

Todos os tratamentos testados apresentaram efeito significativo na eficiência de controle com 7 DAA, indicando a necessidade de maior período de avaliação, para verificar a eficiência do produto. Resultados observados por Abudulai et al. (2003) com produto comercial à base de nim (Neemix) sobre ninfas do percevejo $N$. viridula indicaram mortalidade significativa após 5 dias da aplicação do produto. Em contrapartida, Kamminga et al. (2009), ao testar composto isolado do nim (Aza-Direct), no controle do percevejo Acrosternum hilare (Say, 1831) praga da soja no estado da Virginia (EUA), verificaram que $o$ produto reduziu a população deste percevejo no segundo dia de aplicação, não diferindo do controle no sétimo dia e o mesmo resultado foi observado para adultos de Euschistus servus (Say, 1832). Assim, acredita-se que o isolamento deste composto potencializou a ação inseticida, e isso reduziu o tempo de atividade do produto, indicando sua rápida degradação.

Tabela 1. Médias ( \pm EP) da população de E. heros e eficiência de controle com 2, 5 e 7 dias após a aplicação (DAA) dos tratamentos em condições de campo

\begin{tabular}{cccccccc}
\hline Tratamentos & Prévia $^{\mathbf{n}}$ & $\mathbf{2 ~ D D A}^{\mathbf{n s}}$ & $\mathbf{E \%}^{\mathbf{1}}$ & $\mathbf{5 ~ D D A}^{\mathbf{n s}}$ & $\mathbf{E \%}^{\mathbf{1}}$ & $\mathbf{7 ~ D D A}^{\mathbf{2}}$ & $\mathbf{E \%}^{\mathbf{1}}$ \\
\hline Extrato A. coriacea & $3,0 \pm 0,35$ & $4,6 \pm 0,65$ & 8,91 & $3,4 \pm 0,73$ & - & $3,7 \pm 0,35 \mathrm{~b}$ & 26,73 \\
Extrato A. crassiflora & $2,4 \pm 0,21$ & $4,1 \pm 0,93$ & 17,82 & $3,0 \pm 0,29$ & 13,04 & $4,2 \pm 0,32 \mathrm{~b}$ & 16,83 \\
Controle 1- DMSO 20\% & $3,1 \pm 0,48$ & $5,0 \pm 0,42$ & - & $3,4 \pm 0,49$ & - & $5,0 \pm 0,28 \mathrm{a}$ & - \\
Produto à base de Nim & $3,9 \pm 0,92$ & $5,9 \pm 0,66$ & - & $3,0 \pm 0,35$ & - & $3,9 \pm 0,28 \mathrm{~b}$ & 24,76 \\
Controle 2- Água & $4,1 \pm 0,93$ & $4,4 \pm 0,38$ & - & $2,8 \pm 0,55$ & - & $5,2 \pm 0,45 \mathrm{a}$ & - \\
\hline F & 1,187 & 1,211 & - & 0,336 & - & 3,918 & - \\
$P$ & 0,447 & 0,347 & - & 0,850 & - & 0,023 & - \\
\hline
\end{tabular}

${ }^{\mathrm{NS}}=$ não significativo. ${ }^{1} \% \mathrm{E}=$ Porcentagem de eficiência calculada pela fórmula de Abbott. ${ }^{2}$ Médias \pm erro padrão seguidas da mesma letra na coluna não diferem entre si ao nível de 5\% de probabilidade. 
$\mathrm{Na}$ presente pesquisa foi verificado que a eficiência do extrato de $A$. coriacea foi superior a eficiência de controle de $A$. crassiflora e do produto comercial à base de nim. Este resultado corrobora aos observados em pesquisa com ninfas de $D$. melacanthus e E. heros em laboratório, com maior potencial de controle de A. coriacea em relação a $A$. crassiflora e $A$. dioica (dados não publicados). Também foi verificado que a eficiência de controle para os fitoinseticidas em campo foi menor quando comparados a inseticidas comumente utilizados para o controle de percevejos, com eficiência acima de $90 \%$ aos 3, 6 e 10 dias após a aplicação (DEGRANDE et al., 2000). No entanto, cabe ressaltar que estes extratos não passaram por nenhum processo de isolamento e identificação de moléculas bioativas, $\log$ o são promissores para o controle deste inseto, bem como para o desenvolvimento de pesquisa para novos fitoinseticidas.

\section{CONCLUSÕES}

Os produtos testados apresentaram eficiência de controle a partir de 7 dias após aplicação, e isso indica a necessidade de pesquisas complementares que avaliem estes produtos por maior período.

\section{AGRADECIMENTOS}

A Capes, pela concessão de bolsa de estudos mestrado; ao $\mathrm{CNPq}$, pelo apoio financeiro para realização da pesquisa; Ao grupo Franciosi pela disponibilidade da área de soja, para execução da pesquisa.

\section{REFERÊNCIAS BIBLIOGRÁFICAS}

ABBOTT, W.S. 1925. A method of computing the effectiveness of an insecticide. Journal of Economic Entomology, v.18, p.265-266.

ABUDULAI, M.; SHEPARD, B.M.; SALIFU, A.B. 2003. Field evaluation of a neem (Azadirachta indica A. Juss)based formulation Neemix against Nezara viridula (L.) (Hemiptera: Pentatomidae) in cowpea. International Journal of Pest Management. v.49, n.2, p.109-113.

ALALI, F.Q.; LIU, X.X.; MCLAUGHLIN, J.L. 1999. Annonaceous acetogenins: recent progress. Journal of Natural Products. v.62, n.3, p.504-40.

BATISTA FILHO, A.; RAMIRO, Z.A.; ALMEIDA， J.E.M.; LEITE， L.G.; CINTRA, E.R.R.; LAMAS, C. 2003. Manejo integrado de pragas em soja: impacto de Inseticidas sobre inimigos naturais. Arquivos do Instituto Biológico. v.70, n.1, p.61-67.

BUENO, A.F.; BATISTELA, M.J.; BUENO, R.C.O.F.; FRANÇA-NETO, J.B.; NISHIKAWA, M.A.N.; FILHO, A. L. 2011. Effects of integrated pest management, biological control and prophylactic use of insecticides on the management and sustainability of soybean. Crop Protection, v. 30, n. 7, p. 937-945.

CAVALCANTE, G.M.; MOREIRA, A.F. C.; VASCONCELOS, S.D. 2006. Potencialidade inseticida de extratos aquosos de essências florestais sobre mosca-branca. Pesquisa Agropecuária Brasileira, v.41, n.1, p.9-14.

CORRÊA - FERREIRA, B.S.; ALEXANDRE, T.M.; PELLIZZARO, E.C.; MOSCARDI, F.; BUENO, A.F. 2010. Práticas de manejo de pragas utilizadas na soja e seu impacto sobre a cultura. Londrina: Embrapa soja, 16 p. (Circular Técnica n. ${ }^{\circ}$ 78). Disponível em: http://www.cnpso.embrapa.br/download/ CT78VE.pdf Acesso em 14 dez. 2012.

COSTA, E.L.N.; SILVA, R.F.P.; FIUZA, L.M. 2004. Efeitos, aplicações e limitações de extratos de plantas inseticidas. Acta Biológica Leopoldensia, v.26, n.2, p.173-185. 
DEGRANDE, P.E.; OLIVEIRA, M.A.; SHIMORIHO, A.; BARROS, R. 2000. Controle químico do percevejo Euschistus heros (Fabr., 1794) (Heteroptera: Pentatomidae) na cultura da soja em aplicação aérea. Revista Brasileira de Agrociência v.6, n.2, p.144-148

JACOBSON, M. Botanical pesticides: past, present and future. 1989. In: ARNASON, J.T.; PHILOGENE, B.J.R.; MORAND, P. (Ed.). Insecticides of plant origin. Washington: American Chemical Society. p. 221.

KAMMINGA, K.L.; AMES HERBERT JR, D.; KUHAR, T.P.; MALONE, S.; DOUGHTY, H. 2009. Toxicity, Feeding Preference, and Repellency Associated with Selected Organic Insecticides against Acrosternum hilare and Euschistus servus (Hemiptera: Pentatomidae). Journal of Economic Entomology, v.102, n.5, p.1915-1921.

OLIVEIRA, A.G.; PEREIRA, M. J. B. 2009. Efeito antialimentar do extrato metanólico de Annona crassiflora Mart. sobre o percevejo marrom Euschistus heros (Fabr. 1798) (Heteroptera: Pentatomidae). Revista Brasileira de Agroecologia, v.4. n.2. p.2920-2923.

PANIZZI, A. R.; BUENO, A. F.; SILVA, F. A. C. 2012. Insetos que atacam vagens e grãos. In: HOFFMANN-CAMPO, C. B.; CORRÊA-FERREIRA, B. S.; MOSCARDI, F. (ed.) Soja: manejo integrado de insetos e outros artrópodes-praga. Brasília: Embrapa, $859 \mathrm{p}$.

PANIZZI, A. R.; SLANSKY JÚNIOR, F. 1985. Review of phytophagous pentatomids (Hemiptera: Pentatomidae) associated with soybean in the Americas. Florida Entomology, v.68, n.1, p.184214.

PERES, W.A.A.; CORRÊA-FERREIRA, B. S. 2006. Potencial do óleo de Nim como inseticida vegetal no controle dos percevejos-pragas da soja (Hemiptera: Pentatomidae), Revista Brasileira de Agroecologia, v.1, n.1, p.1651-1655.

RAMIRO, Z.A.; BATISTA FILHO, A.; CINTRA, E.R.R. 2005. Eficiência do inseticida actara mix $110+220$ ce (Thiamethoxam + Cipermetrina) no controle de percevejos pragas da soja, Arquivos do Instituto Biológico, v.72, n.2, p.239-247.

SEYMOUR, J.; BOWMAN, G.; CROUCH, M. 2005. Effects of a neem seed extract on feeding frequency of Nezara viridula (L.) (Hemiptera: Pentatomidae) on pecan nuts. Journal Australian Entomology Society, v.34, p.221-223.

SOSA-GOMEZ, D.R. CORSO, I.C.; MORALES, L. 2001. Insecticide resistance to Endosulfan, Monocrotophos and Metamidophos in the neotropical brown stink bug, Euschistus heros (F.).Neotropical Entomology, v.30, n.2, p.317-320.

SOUZA, E.M.; CORDEIRO, J.R.; PEREIRA, M.J.B. 2007. Avaliação da atividade inseticida dos diferentes extratos das sementes de Annona coriacea sobre Dichelops melacanthus (Dallas, 1851). Revista Brasileira de Agroecologia, v. 2, n. 2, p.1107-1110.

VIVAN, L.M.; DEGRANDE, P.E. 2011. Pragas da soja. In: Fundação MT. Boletim de Pesquisa de Soja, v.11, p.239-297.

Recebido em: 22/02/2013 Aceito para publicação em: 29/11/2013 